\title{
Liquid scintillator composition optimization for use in ultra-high energy cosmic ray detector systems
}

\author{
Dmitriy Beznosko ${ }^{\text {a }}$, Ayan Batyrkhanov, Alexander Iakovlev, and Khalykbek Yelshibekov \\ Nazarbayev University, Physics Department, Astana, Kazakhstan
}

\begin{abstract}
The Horizon-T (HT) detector system and the currently under R\&D HT-KZ detector system are designed for the detection of Extensive Air Showers (EAS) with energies above $\sim 10^{16} \mathrm{eV}\left(\sim 10^{17} \mathrm{eV}\right.$ for HT-KZ). The main challenges in both detector systems are the fast time resolutions needed for studying the temporary structure of EAS, and the extremely wide dynamic range needed to study the spatial distribution of charged particles in EAS disks. In order to detect the low-density of charged particles far from the EAS axis, a large-area detector is needed. Liquid scintillator with low cost would be a possible solution for such a detector, including the recently developed safe and low-cost water-based liquid scintillators. Liquid organic scintillators give a fast and high light yield (LY) for charged particle detection. It is similar to plastic scintillator in properties but is cost effective for large volumes. With liquid scintillator, one can create detection volumes that are symmetric and yet retain high LY detection. Different wavelength shifters affect the scintillation light by changing the output spectrum into the best detection region. Results of the latest studies of the components optimization in the liquid scintillator formulae are presented.
\end{abstract}

\section{Introduction}

In large-area distributed detectors for ultra-high energy cosmic rays, such as the Horizon-T detector system [1] and the HorizonT-KZ (HT-KZ) detector system [2] (currently under development), there is always a question about a medium for charged particle detection. One can choose water and detect Cherenkov light; it is a very fast method but produces low numbers of photons per passing particle. Another choice is an organic scintillator [3], that can be in the form of plastic or liquid scintillator (LS).

An important part of either the plastic or LS is the right choice of fluor and wavelength shifter, and their concentrations. The choice affects the spectral distribution of the light output and the detection efficiency as each photodetector has its own spectral sensitivity region. In this study, the 1,2,4-Trimethylbenzene, or Pseudocumene (PC) based LS was used together with PPO (2,5-diphenyloxazole) as a fluor and MSB (1,4Bis(2-mehylstyryl)benzene) as a wavelength shifter. Both the total light yield (LY) and the spectral differences in the outputs with different amounts of components were measured. This study can be applied for plastic scintillators as well as for the liquid ones. For large-volume detectors planning to use LS with high particles density signals, LY and cost can be reduced by using the novel water-based liquid scintillator material [4].

\section{Fluor concentration optimization}

The schematics for the setup used to measure the LY at different PPO concentrations is shown in Fig. 1. The sample is viewed by two MELZ-FEU [5] PMT-115M

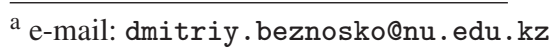

connected to the CAEN [6] DT5743 ADC. The ADC internally handles the double coincidence between the PMT signals. ${ }^{60} \mathrm{Co}$ was used with each sample as the source of radiation. All measurements were conducted inside the black box. PC+PPO solution was used with this setup. A sample histogram of the light intensity for a measurement with $1 \mathrm{~g} / \mathrm{L}$ PPO concentration in the sample is shown in Fig. 2.

The results of the analysis of light intensity dependence on PPO concentration are presented in Fig. 3. From the figure, we can see that that there is no optimal concentration for PC + PPO solution - the dependence is logarithmic and this function increases with the smoothly decreasing slope. The sample with a concentration of $2 \mathrm{~g} / \mathrm{L}$ PPO was chosen for future tests.

After completion of the PPO tests, the wavelength shifter was added to observe how different concentrations will affect the LY. The addition of MSB to the sample caused a decrease in the total amount of light detected by the PMTs. This outcome could be only explained by the spectral sensitivity characteristics of the MELZ-FEU PMT. It has a very uniform sensitivity between $\sim 380 \mathrm{~nm}$ and $\sim 410 \mathrm{~nm}$, and in the first approximation the MSB is shifting the emitted by PPO light (peak at $\sim 380 \mathrm{~nm}$ ) within this range of wavelengths (to $\sim 410 \mathrm{~nm}$ ) with some finite efficiency. This observation has warranted detailed studies of the spectral distributions of LS light to see the actual changes and access the MSB efficiency.

\section{Spectroscopy and MSB contribution}

Sample spectra for different concentrations of shifter in the solution were obtained. Measurements were done using an Agilent Cary Eclipse spectrophotometer [7]. In order to preserve the output spectra of the PPO as seen by the 


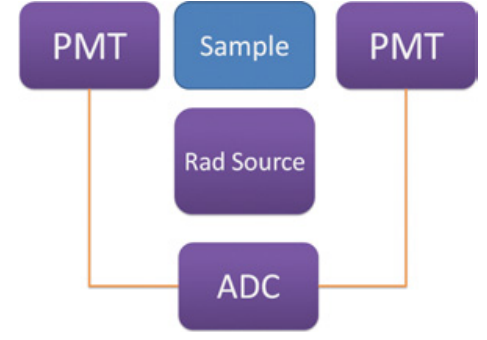

Figure 1. Experimental setup schematics for PPO concentration optimization.

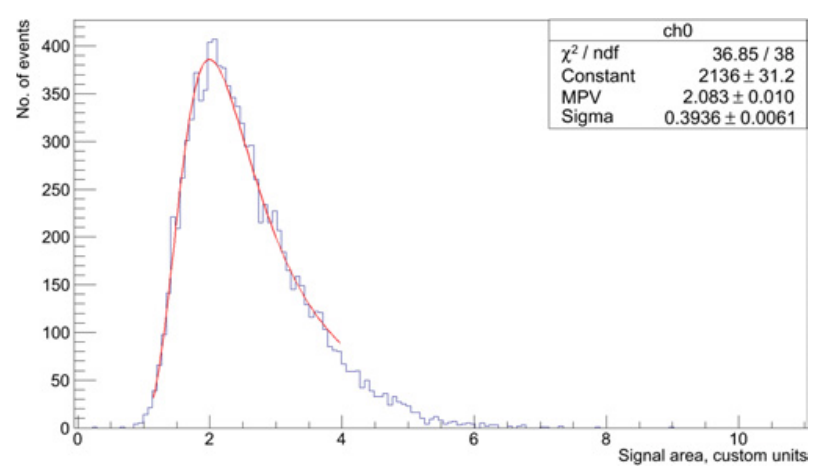

Figure 2. Sample data for $1 \mathrm{~g} / \mathrm{L}$ PPO concentration.

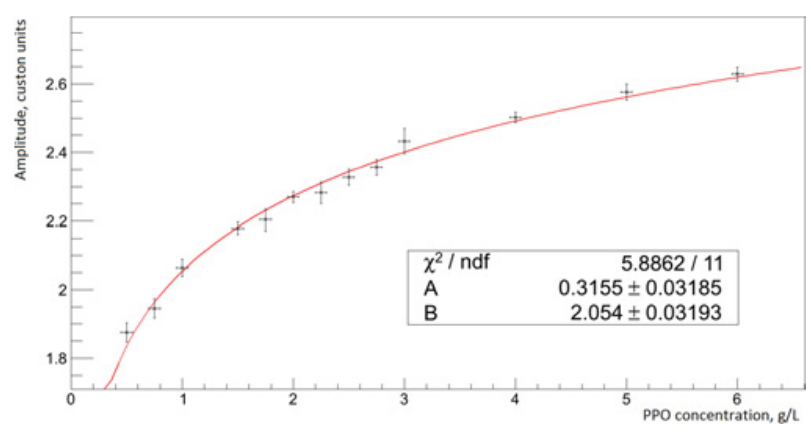

Figure 3. Light yield vs. PPO concentration.

shifter and minimally affect the added shifter directly, the wavelength of the excitation light was chosen at $275 \mathrm{~nm}$ that corresponds to MSB minimal absorption so that it will be absorbed by PPO only. The read-out range of the emitted light was chosen between $300-550 \mathrm{~nm}$.

The output of the LS with only PPO added is presented in Fig. 4. Changes in the shape of the spectra with MSB concentrations of $8 \mathrm{mg} / \mathrm{L}$ can be seen in Fig. 5 . Data is fitted by the empirical equation to determine the contribution of each component. $\mathrm{PPO}, \mathrm{MSB}_{e m}, \mathrm{MSB}_{e x}$ and $\mathrm{PC}_{\text {pure }}$ are the parameters for light emitted by PPO, MSB absorption of PPO light, MSB re-emission, and PC/other background contributions. The parameters of most interest are $\mathrm{MSB}_{e x}$ and $\mathrm{MSB}_{\text {em }}$ that represent the light absorbed and emitted by MSB. The ratio of these two parameters shows that only about $50 \%$ of the light is re-emitted. Thus, integral LY is reduced.

\section{WbLS testing}

WbLS potentially combines both the Cerenkov ring and scintillation light capabilities. It can preserve the particle identification for particles above the Cerenkov threshold,

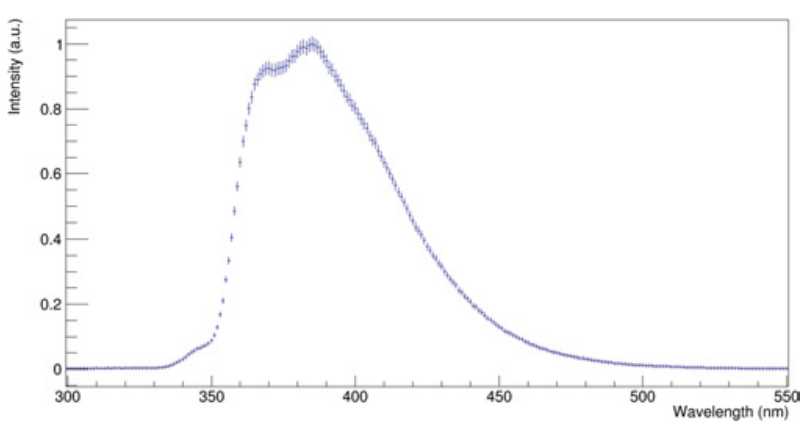

Figure 4. LS + PPO output spectrum.

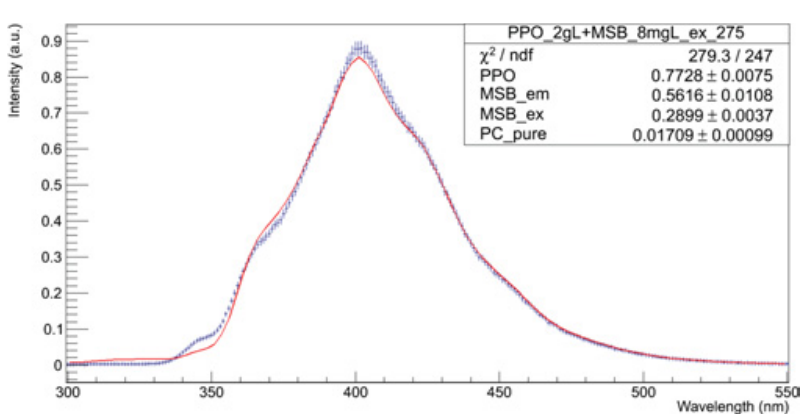

Figure 5. Output spectrum with MSB concentration of $8 \mathrm{mg} / \mathrm{L}$.

and detect the charged particles below the threshold via scintillation light. In addition, WbLS features the lower cost than pure LS and it is safer to handle [4]. For high energy cosmic rays, WbLS can be a medium with a tunable LY. The ability to reach the desired LY can be checked using a mono-energetic proton beam with different WbLS concentrations. For the test, two different WbLS formulations $(0.4 \%$ and $0.99 \%)$, pure water and pure LS samples were chosen. Three different proton beam energies were used with each sample. The choice of the energies comes from the following considerations:

- $2000 \mathrm{MeV}$ protons behave as minimum ionizing particle (MIP)

- $475 \mathrm{MeV}$ protons are just below the Cerenkov limit in water

- $210 \mathrm{MeV}$ protons have $\sim$ same energy deposition as $\mathrm{K}^{+}$from the proton decay simulation.

The details of the experimental setup and measurements can be found in [4]. A surfactant (Linear AlkylBenzene Sulfonic Acid, LAS) is used to dissolve the PC, a common LS material also used for WbLS, in water. The PPO and MSB are used as a fluor and a wavelength shifter to downshift the ultraviolet light from the LS into the blue region of the spectrum where water has higher transparency.

The composition of the 4 samples tested in this experiment:

- Water (purified)

- WbLS-1: $0.4 \%$ PC + 0.4 g/L PPO + $3 \mathrm{mg} / \mathrm{L}$ $\mathrm{MSB}+$ surfactant in water

- WbLS-2: $0.99 \% \mathrm{PC}+1.36 \mathrm{~g} / \mathrm{L} \mathrm{PPO}+7.48 \mathrm{mg} / \mathrm{L}$ $\mathrm{MSB}+$ surfactant in water

- LS: PC + 2 g/L PPO + 15 mg/L MSB. 


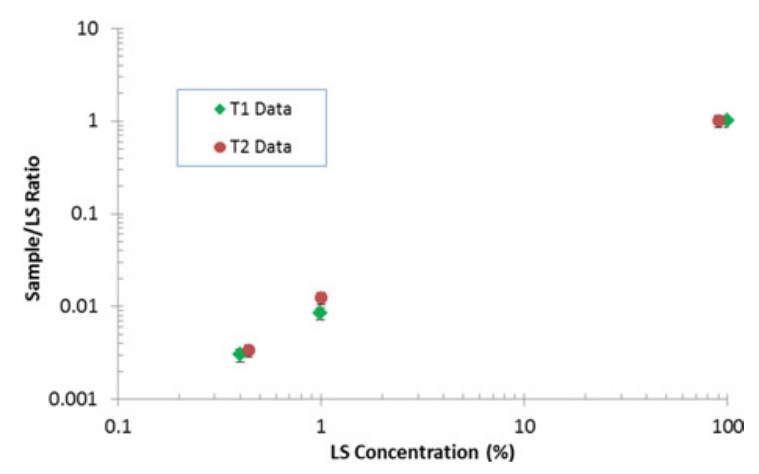

Figure 6. WbLS output ratio to LS for $475 \mathrm{MeV}$ proton energy. Some points are shifted for visibility.

The water sample is used to control systematics and to subtract the Cherenkov light contribution to the scintillation light. The result for the two test volumes (T1 and T2) at $475 \mathrm{MeV}$ proton beam energy is shown in Fig. 6 as WbLS LY ratio to pure LS. The results for other energies is similar.

The LY for pure LS and two formulations of the WbLS have been successfully measured. The $0.99 \%$ WbLS sample yields $\sim 1 \%$ light of the pure LS; assuming that typical LY of LS is 10000 optical photons per $\mathrm{MeV}$ suggests 100 photons/MeV for this concentration. Therefore, the WbLS that satisfies the requirements for different detector needs can be fabricated. The result also illustrates that different LY can be easily achieved by adjusting the WbLS components concentration. Additional work is planned at Nazarbayev University to test the LS optimization with WbLS.

\section{Conclusion}

Work has been done on the optimization of LS composition. The results can also be applied to plastic scintillators as well. The concentration of each LS component can be chosen based on the needs from the fit parameters of the LY. Experiment showed that the amount of output light is reduced when the shifter is added to base + fluor solution as shifter efficiency is $\sim 50 \%$. The choice of the wavelength shifter depends on the sensitivity of the detector used for the experiment. This study plans to see the results applicability to WbLS and continue the optimization tests.

The project is financially supported by the grant of the Corporate Fund "Fund of Social Development"

\section{References}

[1] RU Beisembaev et al., arXiv:1605.05179 (2016)

[2] A. Duspayev et al., in proceedings to PhotoDet2015 conference, PoS(PhotoDet2015)056, (2015)

[3] A Baitenov et al., arXiv:1601.00086 (2016)

[4] L. J. Bignell et al., Journal of Instrumentation, 10, 12009 (2015)

[5] MELZ-FEU, 4922-y pr-d, 4c5, Zelenograd, g. Moskva, Russia, 124482 http: //www.meltz-feu.ru

[6] CAEN S.p.A. Via della Vetraia, 11, 55049 Viareggio Lucca, Italy, http: //www . caen. it

[7] Agilent INC. 5301 Stevens Creek Blvd, Santa Clara, CA 95051, US, http://agilent.com 